\title{
Roles of Conserved Ectodomain Cysteines of the Rat P2X4 Purinoreceptor in Agonist Binding and Channel Gating
}

\author{
M. B. ROKIC ${ }^{1}$, V. TVRDOŇOVÁ ${ }^{1}$, V. VÁVRA ${ }^{1}$, M. JINDŘICHOVÁ ${ }^{1}$, T. OBŠIL ${ }^{2}$, \\ S. S. STOJILKOVIC ${ }^{3}$, H. ZEMKOVÁ ${ }^{1}$
}

${ }^{1}$ Department of Cellular and Molecular Neuroendocrinology, Institute of Physiology of the Academy of Sciences of the Czech Republic, Prague, Czech Republic, ${ }^{2}$ Department of Physical and Macromolecular Chemistry, Faculty of Science, Charles University, Prague, Czech Republic, ${ }^{3}$ Section on Cellular Signaling, Program in Developmental Neuroscience, NICHD, NIH, Bethesda, USA

Received February 17, 2010

Accepted April 16, 2010

On-line April 20, 2010

\begin{abstract}
Summary
Mammalian P2X receptors contain 10 conserved cysteine residues in their ectodomains, which form five disulfide bonds (SS1-5). Here, we analyzed the relevance of these disulfide pairs in rat P2X4 receptor function by replacing one or both cysteines with alanine or threonine, expressing receptors in HEK293 cells and studying their responsiveness to ATP in the absence and presence of ivermectin, an allostenic modulator of these channels. Response to ATP was not altered when both cysteines forming the SS3 bond (C132-C159) were replaced with threonines. Replacement of SS1 (C116-C165), SS2 (C126-C149) and SS4 (C217-C227), but not SS5 (C261-C270), cysteine pairs with threonines resulted in decreased sensitivity to ATP and faster deactivation times. The maximum current amplitude was reduced in SS2, SS4 and SS5 double mutants and could be partially rescued by ivermectin in SS2 and SS5 double mutants. This response pattern was also observed in numerous single residue mutants, but receptor function was not affected when the 217 cysteine was replaced with threonine or arginine or when the 261 cysteine was replaced with alanine. These results suggest that the SS1, SS2 and SS4 bonds contribute substantially to the structure of the ligand binding pocket, while the SS5 bond located towards the transmembrane domain contributes to receptor gating.
\end{abstract}

\section{Key words}

Purinergic signaling • ATP-gated receptor-channels • Disulfide bonds $\bullet$ Ivermectin $\bullet$ Deactivation

\section{Corresponding author}

H. Zemková, Institute of Physiology, Academy of Sciences of the Czech Republic v.v.i., Vídeňská 1083, 14220 Prague 4, Czech Republic. E-mail: zemkova@biomed.cas.cz. Fax: 420241 062488

\section{Introduction}

P2X receptors (P2XRs) comprise a family of ATP-gated cationic channels expressed in numerous excitable and non-excitable cells, and they play important roles in a variety of physiological processes (Khakh and North 2006). Seven mammalian P2XR subunits, termed P2X1-7, and many non-mammalian subunits have been identified (Jarvis and Khakh 2009, Surprenant and North 2009). The extracellular loop of all mammalian P2X subunits contains 10 conserved cysteine residues that have been predicted to make bonds in the following order (P2X4R numbering): 116-165 (SS1), 126-149 (SS2), 132-159 (SS3), 217-227 (SS4) and 261-270 (SS5) (Clyne et al. 2002, Ennion and Evans 2002). Direct evidence for the existence of these disulfide bonds was provided by solving the crystal structure of zebrafish P2X4.1R (Kawate et al. 2009) (Fig. 1A).

At the present time, it is less clear what is the role of these bonds in receptor functions. The disulfide bonds in the human $\mathrm{P} 2 \mathrm{X} 1 \mathrm{R}$ and rat $\mathrm{P} 2 \mathrm{X} 2 \mathrm{R}$ are not responsible for the formation of multimers (Rassendren et 
al. 1997, Clyne et al. 2002, Ennion and Evans 2002). The cysteine residues forming $\mathrm{SS} 2$ and $\mathrm{SS} 3$ bonds together with a nearby histidine residue may contribute to the formation of a metal ion-binding site of P2X2R (Clyne et al. 2002, Friday and Hume 2008) and P2X4R (Coddou et al. 2007). Disruption of the SS5 disulfide bond in the human P2X1R by point mutations decreased expression of the mutated receptor at the cell surface, suggesting that this bond could be involved in trafficking of the receptor to the cell membrane (Ennion and Evans 2002). The relevance of $\mathrm{SS}$ bonds in responsiveness of $\mathrm{P} 2 \mathrm{X} 4 \mathrm{R}$ to ethanol was also suggested (Yi et al. 2009). Several cysteine-to-alanine point mutants of P2X1R, P2X2R and $\mathrm{P} 2 \mathrm{X} 4 \mathrm{R}$ expressed in oocytes also show decreased sensitivity to ATP or were non-functional depending on the receptor subtypes. Furthermore, the ectodomain of P2XR in Ostreococcus tauri probably forms only one bond (SS1) and shows a low sensitivity to ATP (Surprenant and North 2009), raising the possibility that SS bonds may also contribute to agonist binding and/or channel gating.

To study the role of conserved cysteine residues in receptor function, we generated five double mutants of the rat $\mathrm{P} 2 \mathrm{X} 4 \mathrm{R}$ using threonine as a substitute for cysteine pairs, as well as single residue mutants by replacing conserved cysteines with threonine or alanine. As a receptor model, we purposely selected P2X4R because of two reasons. First, the sensitivity of rat P2X4R to ivermectin (IVM) (Khakh et al. 1999, Priel and Silberberg 2004, Jelinkova et al. 2006, Toulme et al. 2006, Silberberg et al. 2007) provides an effective tool for studying low functioning mutants (Zemková et al. 2007). Second, the recently crystallized zebrafish P2X4.1 receptor (Kawate et al. 2009) offered a template for mechanistic explanations of the role of SS bonds in mammalian P2X4Rs.

\section{Materials and Methods}

\section{Cells culture and transfection}

Experiments were performed in HEK293 cells (American Type Culture Collection), which were grown in Dulbecco's modified Eagle's medium supplemented with $10 \%$ fetal bovine serum, $50 \mathrm{U} / \mathrm{ml}$ penicillin and $50 \mu \mathrm{g} / \mathrm{ml}$ streptomycin in a humidified $5 \% \mathrm{CO}_{2}$ atmosphere at $37{ }^{\circ} \mathrm{C}$. Transfection of either wild type (WT) or mutant receptors was conducted using $2 \mu \mathrm{g}$ of DNA and $5 \mu$ of Lipofectamine 2000 in $2 \mathrm{ml}$ of serumfree Opti-MEM according to the manufacturer's instructions (Invitrogen, UK or Carlsbad, CA, USA) or $2 \mu \mathrm{g}$ of DNA and $4 \mu \mathrm{l}$ of jetPRIME ${ }^{\mathrm{TM}}$ reagent in $2 \mathrm{ml}$ of Dulbecco's modified Eagle's medium according to manufacturer's instructions (PolyPlus-transfection, Illkirch, France).

\section{DNA constructs}

cDNAs encoding the sequences of the rat P2X4R and mutated subunits were subcloned into the biscistronic enhanced fluorescent protein expression vector pIRES2-EGFP (Clontech, Mountain View, CA, USA). Mutagenesis (QuikChange site-directed mutagenesis kit; Stratagene, La Jolla, CA) was performed using $\mathrm{P} 2 \mathrm{X}$ 4/pIRES2-EGFP as the template with oligonucleotides (synthesized by VBC-Genomics, Vienna, Austria) introducing specific point mutations. The C-terminal EGFP-tagged P2X4 construct was also used for mutagenesis (Yan et al. 2005). The presence of the mutations and the identity of all constructs were verified by dye terminator cycle sequencing (ABI PRISM 3100, Applied Biosystems, Foster City, CA - performed by the Laboratory of DNA sequencing, Institute of Microbiology, ASCR, Prague).

\section{Localization of EGFP-tagged receptors}

The localization of EGFP-tagged P2X4Rs in cells was examined by laser scanning confocal microscopy (Leica SP2 AOBS, Germany). Images were collected under a 60x objective lens with further zoom applied.

\section{Patch clamp recordings}

Currents were recorded in a whole-cell configuration from cells clamped to $-60 \mathrm{mV}$ using an Axopatch 200B patch-clamp amplifier (Axon Instruments, Union City, CA). Recordings were captured and stored using the Digidata 1322A and pClamp9 software package During the experiments, the cell culture was continuously perfused with an extracellular solution containing (in mM): $142 \mathrm{NaCl}, 3 \mathrm{KCl}, 2 \mathrm{CaCl}_{2}, 1 \mathrm{MgCl}_{2}$, 10 HEPES and 10 D-glucose, adjusted to $\mathrm{pH} 7.3$ with $1 \mathrm{M} \mathrm{NaOH}$. Patch electrodes used for whole-cell recording were filled with an intracellular solution containing (in mM): $154 \mathrm{CsCl}$, EGTA 11 and HEPES 10, with $\mathrm{pH}$ adjusted to 7.2 with $1 \mathrm{M} \mathrm{CsOH}$. Cells were examined before and 4-6 min after treatment with $3 \mu \mathrm{M}$ ivermectin. Control and ATP-containing solutions were applied using an RSC-200 Rapid Solution Changer system (BIO-LOGIC, Claix, France). 


\section{Calculations}

Dose-response data points were fitted by equation: $y=I_{\max } /\left(1+\left(E C_{50} / x\right)^{n H}\right.$, where $y$ is the amplitude of the current evoked by ATP; $I_{\max }$ is the maximum current amplitude induced by $100 \mu \mathrm{M}$ ATP; $\mathrm{EC}_{50}$ is the agonist concentration producing $50 \%$ of the maximal response; $n_{H}$ is the Hill coefficient; and $x$ is the concentration of ATP; (SigmaPlot 2000 v9.01; SPSS Inc., Chicago, IL). For clarity, we used $n_{H}=1.3$ for all mutants; this value was obtained by fitting the wild type receptor dose response curve. The kinetics of current decay evoked by washout of the agonists, termed deactivation, were fitted by a single exponential function $\left[y=A \exp \left(-t / \tau_{\text {off }}\right)\right]$ using the program CLAMPFIT 9 (Axon Instruments), where $A$ is the amplitude of current, and $\tau_{\text {off }}$ is the deactivation time constant. All data are reported as mean \pm S.E.M. Significant differences between means were determined by one-way analysis of variance using SigmaStat 2000 v9.01 and Tukey's post hoc test, $\mathrm{P}<0.01$.

\section{Results}

To study the functional roles of 10 conserved cysteine residues forming disulfide bonds in the ectodomain of the rat $\mathrm{P} 2 \mathrm{X} 4 \mathrm{R}$ (Fig. 1A), these residues were substituted with alanine (A-mutants) or threonine (T-mutants). Figure 1B shows no obvious difference in the cellular distribution of EGFP-tagged WT and A-mutants of the P2X4R. In all cases, the EGFP fluorescence was predominantly localized in the plasma membrane region. The responsiveness of WT and A-, T-mutants to ATP was analyzed in the presence and absence of $3 \mu \mathrm{M}$ IVM using patch-clamp whole-cell recording. In cells expressing the WT receptor, IVM treatment caused three types of changes: it increased the sensitivity of receptors to ATP by approximately 8-fold, as indicated by a leftward shift in the $\mathrm{EC}_{50}$ value; it augmented $I_{\max }$ by 1.7 -fold; and it slowed the deactivation kinetics of the receptors by about 60 -fold, as estimated by $\tau_{\text {off }}$ (Fig. 1A, inset).

\section{Cysteine pair mutants}

In initial experiments, we used mutants in which both cysteines were substituted with threonines. The results of these investigations are shown in Figures 2 and 3 (right panels) and summarized in Table 1. The SS1 double mutant showed low sensitivity to ATP, with its $\mathrm{EC}_{50}$ value increased 12-fold compared to that of WT receptor, accompanied by rapid deactivation (Fig. 2A) but no effect on $\mathrm{I}_{\max }$ (Fig. 3). The SS2 double mutant also showed a rightward shift in the sensitivity to ATP $\left(\mathrm{EC}_{50}\right.$ value increased 14-fold) but exhibited a significant decrease in the $I_{\max }$, which was partly rescued with IVM treatment; the increase in the $I_{\max }$ for this mutant was significantly higher (3-fold) than in cells expressing the WT receptor (1.7-fold).

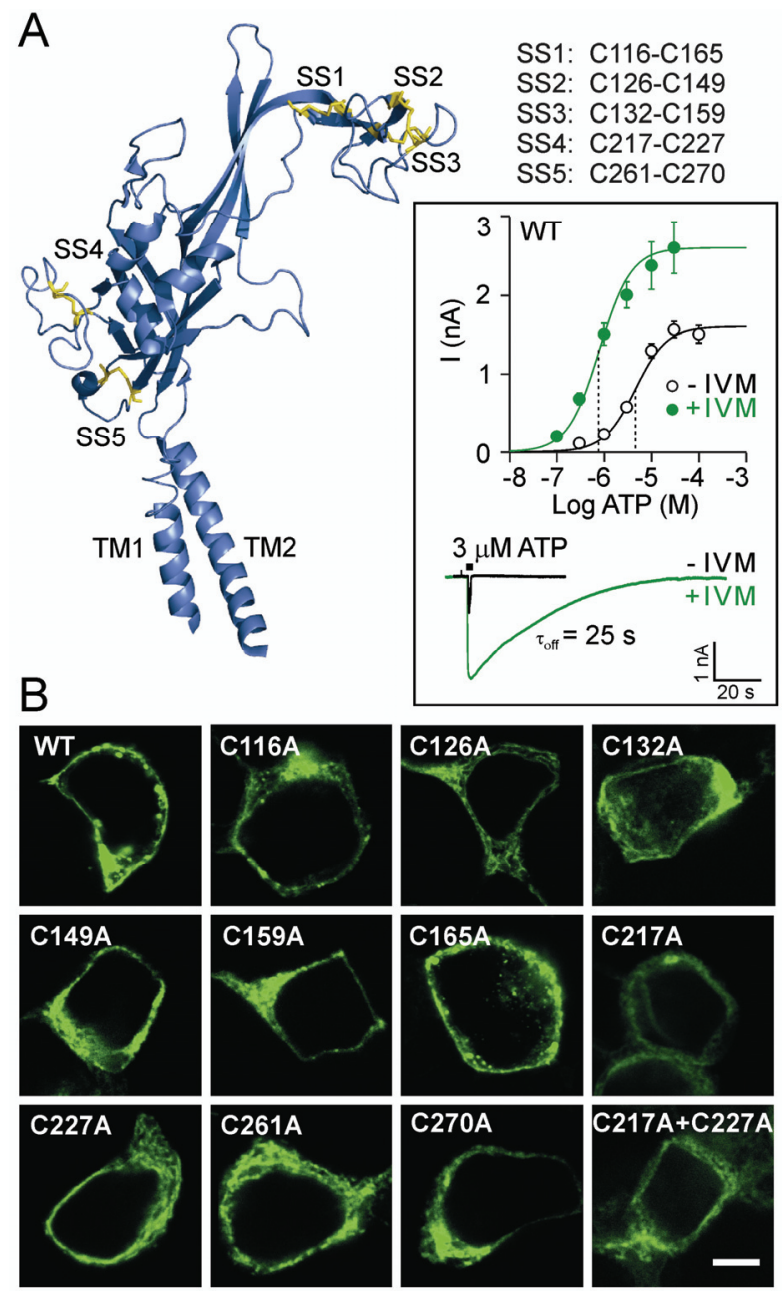

Fig. 1. Expression and function of wild type (WT) and mutant rat P2X4R in HEK293 cells. (A) Secondary structure organization of one zebrafish P2X4.1 receptor subunit (Kawate et al. 2009) with $\alpha$-helices, $\beta$-sheets and turns in blue and disulfide bonds in yellow (PyMol; pdb 3I5D). Five disulfide bonds are formed between the following conserved cysteine residues (rat P2X4R numbering):116-165 (SS1), 126-149 (SS2), 132-159 (SS3), 217227 (SS4) and 261-270 (SS5). (Inset) Potentiating effects of IVM on ATP-induced current in HEK293 cells expressing WT-P2X4R. Upper part: concentration dependence of ATP on the peak current amplitude in the absence (open symbols) and presence (closed symbols) of $3 \mu \mathrm{M}$ IVM. Data points are mean \pm S.E.M. values from 5-17 cells per dose. The vertical dotted lines represent the mean $\mathrm{EC}_{50}$ values. Lower part: a sample recording showing the effects of IVM on the peak amplitude and deactivation time $\left(\tau_{\text {off }}\right)$ of current induced by stimulation with $3 \mu$ M ATP (2-s pulse). The number represents the $\tau_{\text {off }}$ value in the presence of IVM. (B) The expression pattern of the WT receptor, ten conserved cysteine-to-alanine single mutants, and the C217A+C227A double mutant of the rat P2X4R in HEK293 cells. Scale bar: $5 \mu \mathrm{m}$. 
In contrast, replacement of the SS3 cysteines with threonines did not affect receptor function. Double mutation of the SS4 cysteines resulted in a practically non-functional channel for which the $\mathrm{EC}_{50}$ value could not be determined, and the current amplitude was not rescued by IVM. Similar to the SS1 and SS2 double mutants, the deactivation time constant estimated after the removal of agonist in the presence of IVM was significantly reduced in the SS4 double mutant (Fig. 2A), suggesting that its ATP sensitivity was also reduced. The SS5 double mutant also responded with low peak current amplitude. After IVM treatment, however, the current amplitude was enlarged. The increase in $I_{\max }$ for this double mutant was significantly higher (3.5-fold increase) than for the WT receptor, and the $\mathrm{EC}_{50}$ value was shifted slightly leftward (Fig. 3).

\section{Single cysteine mutants}

To evaluate the SS bond-dependent and bondindependent effects of individual cysteine residues in a particular pair, 10 conserved cysteine residues were replaced one by one by threonine $(\mathrm{T})$ or alanine (A). As for the double SS1 mutants, single A- and T-mutants of cysteine 116 and 165 residues showed a significant decrease in the sensitivity of P2X4R to ATP, in both the presence and absence of IVM, without affecting $\mathrm{I}_{\max }$ (Fig. 3 , Table 1). These changes were accompanied by faster receptor deactivation after removal of agonist (Fig. 2B), indicating that disruption of SS1 was solely responsible for the observed decrease in receptor sensitivity to ATP.

In contrast, for the three single point SS2 mutants, C126T, C149A and C149T, the $I_{\max }$ amplitude was much lower compared with the SS2 double and C126A single mutants. In the presence of IVM, the A- and T-mutants of cysteines 126 and 149 also mimicked the effects of the double SS2 mutant on ATP sensitivity; a rightward shift in the $\mathrm{EC}_{50}$ was accompanied by faster deactivation, and the fold-increase of $\mathrm{I}_{\max }$ current for all SS2 single mutants was higher (3- to 7-fold increase) than for the WT receptor.

Receptor function was not obviously affected by the $\mathrm{C} 132 \mathrm{~T}, \mathrm{C} 159 \mathrm{~A}$ and $\mathrm{C} 159 \mathrm{~T}$ mutation, confirming that disruption of SS3 bond alone does not significantly affect P2X4R function. Furthermore, the C132A mutation resulted in the channel responding to ATP with a significantly smaller current, a rightward shift in the $\mathrm{EC}_{50}$ and faster deactivation time compared to WT receptor (Fig. 2B). These effects probably reflect the introduction of alanine at this position rather than liberating the Cys ${ }^{159}$, because the C132T mutant was fully functional.

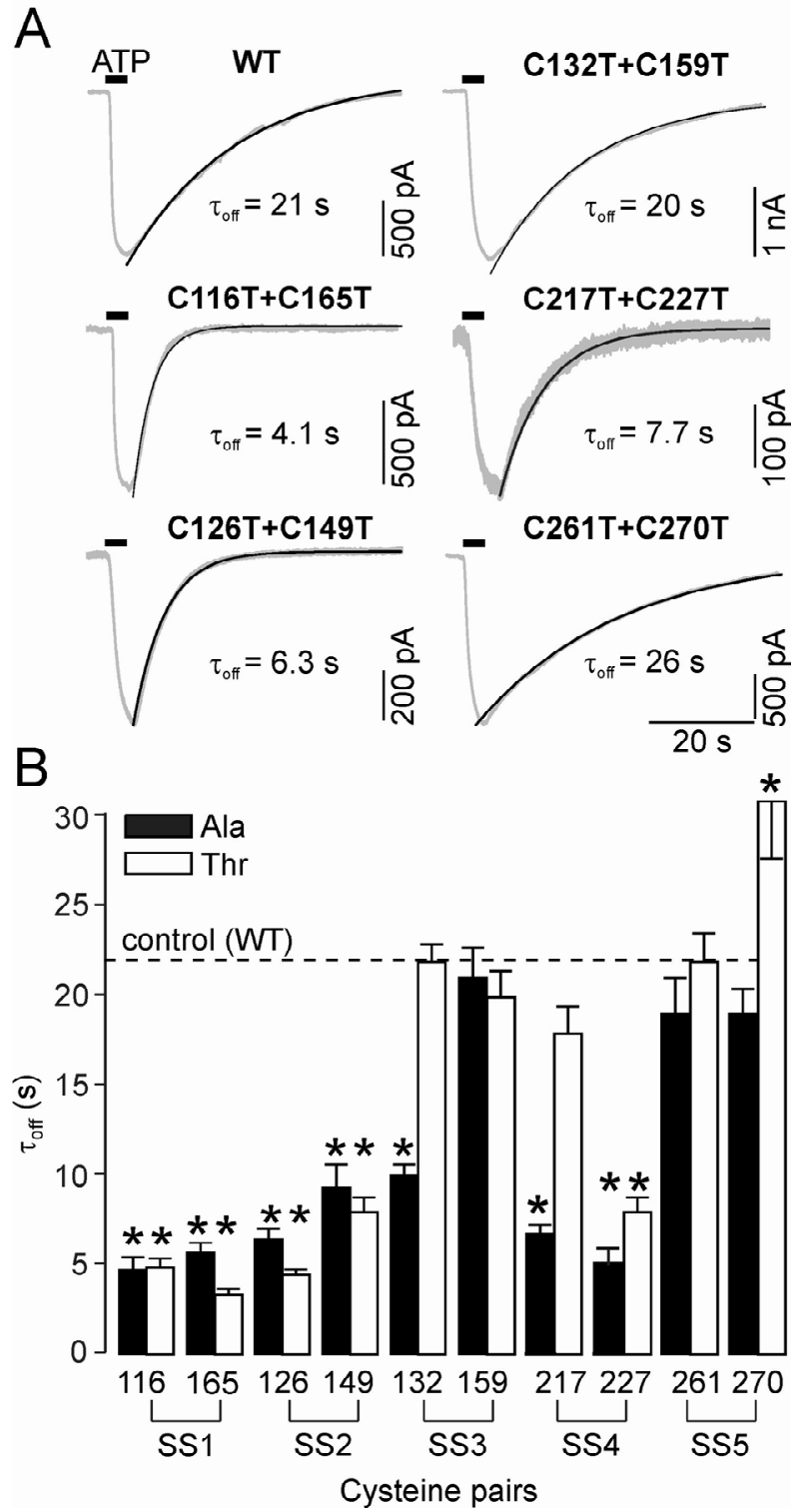

Fig. 2. Effects of the disruption of disulfide bonds on the deactivation kinetics of P2X4R. (A) Sample recordings of ectodomain cysteine double mutants showing differences in the current decay after washout of ATP. All traces were recorded 4-6 min after the application of $3 \mu \mathrm{M}$ IVM, and a nondesensitizing concentration of ATP (1-3 $\mu \mathrm{M}$ for SS1-3 and SS5 and $30 \mu \mathrm{M}$ for SS4) was used for stimulation. The time of ATP application is indicated by horizontal bars. Numbers below the traces indicate the deactivation time constant $\left(\tau_{\text {off }}\right)$ values. (B) Comparison of IVM effects on the deactivation time constant ( $\left.\tau_{\text {off }}\right)$ of A- (closed bars) and T- (open bars) single-point mutants of cysteine residues forming the disulfide bonds SS1-5. $\left.{ }^{*}\right)$ significantly different $(p<0.05)$ from WT value.

The loss of channel function by replacing the SS4 cysteines with threonines was completely mimicked by C217A, C227A and C227T mutations; these mutants showed a low response to ATP in the absence and presence of IVM (Fig. 3, Table 1). In contrast, the peak current amplitude and ATP sensitivity was not 

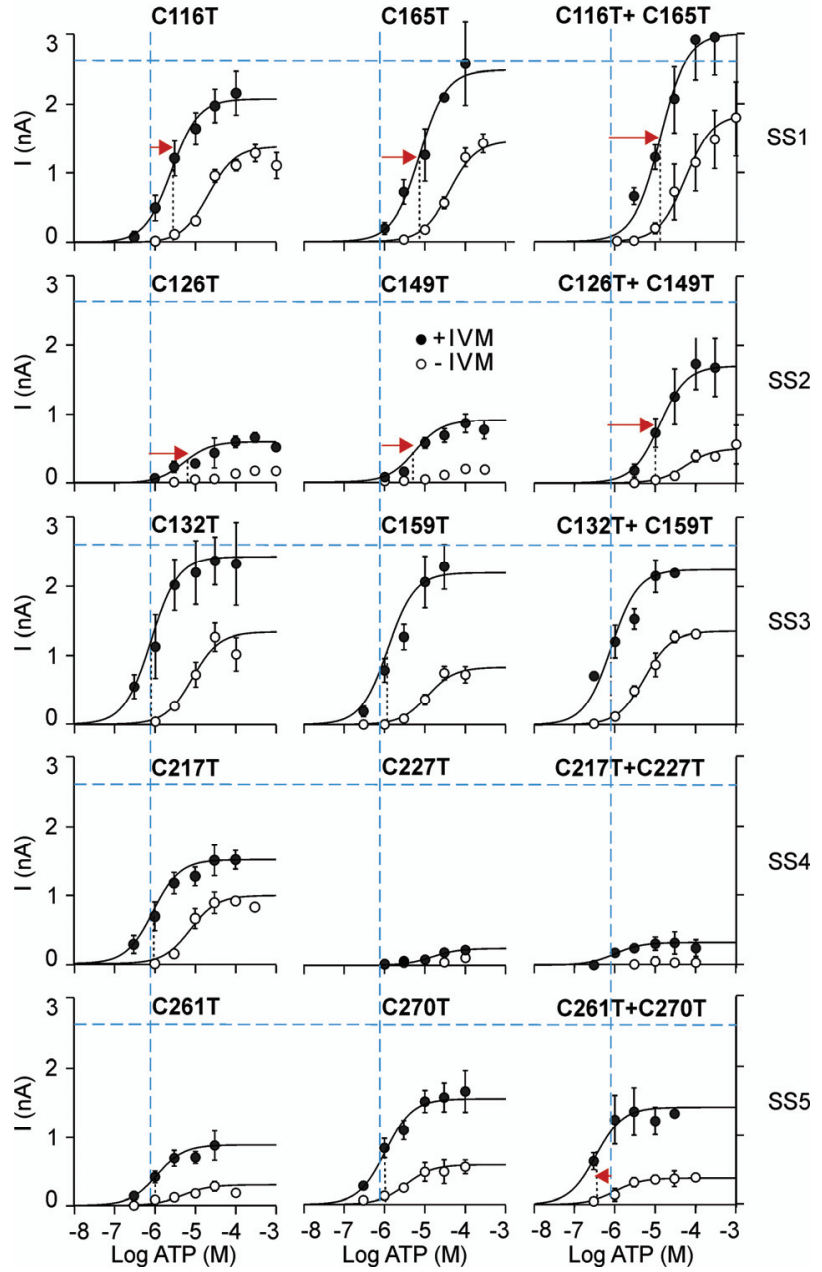

Fig. 3. ATP dose-response curves for single and double threonine mutants of P2X4R. Concentration dependence of ATP on the peak current amplitude responses in single-point mutants (left and middle panels) and double mutants (right panels) for five disulfide bonds is shown in the absence (open circles) and presence (closed circles) of $3 \mu \mathrm{M}$ IVM. Each row shows paired cysteine mutants that form disulfide bonds (SS1-5). Vertical and horizontal dashed lines (in blue) indicate the $\mathrm{EC}_{50}$ and $\mathrm{I}_{\max }$ values, respectively, for P2X4R-WT in the presence of $3 \mu \mathrm{M}$ IVM. The vertical dotted lines (in black) represent the mean $\mathrm{EC}_{50}$ values for T-mutants in the presence of IVM and significant shifts (red arrows). Data shown are mean \pm S.E.M. from 6-30 cells.

significantly affected by replacing cysteine 217 with threonine or arginine, whereas the $\mathrm{C} 217 \mathrm{E}, \mathrm{C} 217 \mathrm{~L}$, and C227R mutants were non-functional (Table 1). As for the double SS4 mutant, the deactivation kinetics, but not the $\mathrm{EC}_{50}$ value, could be determined for $\mathrm{C} 217 \mathrm{~A}, \mathrm{C} 227 \mathrm{~A}$ and C227T mutants in the presence of IVM. The low $\tau_{\text {off }}$ value of these mutants (Fig. 2B) indicated that they have reduced sensitivity to ATP. The deactivation kinetics of the $\mathrm{C} 217 \mathrm{~T}$ and $\mathrm{C} 217 \mathrm{R}$ mutant was comparable to those of WT-P2X4R (Table 1).

A decrease in the peak current amplitude observed in cells expressing the SS5 double mutant was mimicked by single point mutants C261T, C270T and
C270A (Figs 2 and 3) and the stimulatory effect of IVM on $\mathrm{I}_{\max }$ was also higher (3- to 7-fold increase) than for the WT receptor (1.7-fold increase). No changes in the sensitivity to ATP were observed in single SS5 mutants, and the deactivation time constant of the C270T mutant was higher compared to the WT receptor (Fig. 2B). In contrast, the C261A mutant was fully functional, indicating that liberated $\mathrm{Cys}^{270}$ or alanine as a substitute at 261 position rescued the receptor function after disruption of the SS5 bond.

\section{Discussion}

The aim of our study was to understand the role of SS bonding in agonist binding and channel gating using rat $\mathrm{P} 2 \mathrm{X} 4 \mathrm{R}$ as a receptor model. We show that disruption of the SS1, SS2 and SS4 bonds by substituting both cysteines with threonine generated less sensitive receptors. All four SS1 single mutants, three of four SS2 single mutants and six of eight SS4 single mutants showed similar effect. Of these three bonds, mutation of SS4 cysteine residues generated the most profound changes in the receptor function. For SS4 double mutant and six single mutants, the $\mathrm{EC}_{50}$ values could not be determined, but the amplitude of response was sufficient to estimate deactivation time constant in the presence of IVM. Because there is a significant correlation between the $\mathrm{EC}_{50}$ values for $\mathrm{ATP}$ and the rate of receptor deactivation in the presence of IVM for ectodomain mutants (Zemková et al. 2007), it is reasonable to conclude that these SS4 mutants are also less sensitive. This conclusion is in general agreement with the data shown by Evans' and Hume's groups. The P2X1RC217A and the P2X1R-C227A mutants were fully functional but were the least sensitive among 10 A-mutants (Ennion and Evans 2002). The P2X2RC224A mutant expressed in oocytes was not functional, whereas the C214A mutant showed decreased sensitivity to ATP. Furthermore, the $\mathrm{I}_{\max }$ value for both SS4 mutants was significantly reduced or undetectable when P2X2R was expressed in HEK293 cells (Clyne et al. 2002).

We also found that the double SS5 mutant and three of four single SS5 mutants show low $\mathrm{I}_{\max }$ and $\mathrm{EC}_{50}$ values closer to that of the WT receptor. Their rates of deactivation in the presence of IVM were comparable to those observed in controls, clearly indicating that their sensitivity for ATP is not affected. The sensitivity of the SS5 A-mutants of P2X1R and P2X2R was also not affected and $I_{\max }$ values were significantly lower (Clyne et al. 2002, Ennion and Evans 2002). It has been 
Table 1. Characterization of alanine (A), threonine $(T)$ and arginine (R) single mutants and double-point mutants of cysteine pairs SS1-5 in the rat P2X4R ectodomain. The $\mathrm{EC}_{50}$ values represent the ATP concentration producing a half-maximum effect, whereas 100-300 $\mu \mathrm{M}$ ATP was used to estimate $\mathrm{I}_{\max }$ in both the presence and absence of ivermectin (IVM). The deactivation time constant ( $\tau_{\text {off }}$ ) was derived from mono-exponential fitting of the current decay after washout of 3 or $10 \mu \mathrm{M}$ ATP in the presence of $3 \mu \mathrm{M}$ IVM. Each receptor was examined in 6 to 31 cells. Data shown are mean \pm S.E.M.; $(*) p<0.01$ between wild type (WT) and the mutated receptor; n. d., these values could not be determined.

\begin{tabular}{|c|c|c|c|c|c|c|}
\hline Bonds & P2X4R & $\begin{array}{l}-\mathbf{I V M} \\
\mathbf{E C}_{\mathbf{5 0}}(\mu \mathrm{M})\end{array}$ & $\begin{array}{l}+ \text { IVM } \\
\text { EC }_{50}(\mu M)\end{array}$ & $\begin{array}{l}-\mathrm{IVM} \\
\mathbf{I}_{\max } \\
(\mathbf{n A})\end{array}$ & $\begin{array}{l}+ \text { IVM } \\
\text { I }_{\max } \\
(\mathbf{n A})\end{array}$ & $\begin{array}{l}+ \text { IVM } \\
\tau_{\text {off }} \\
(\mathbf{s})\end{array}$ \\
\hline \multirow{6}{*}{$S S 1$} & WT & $4.6 \pm 0.5$ & $0.8 \pm 0.2$ & $1.5 \pm 0.2$ & $2.6 \pm 0.2$ & $22 \pm 0.6$ \\
\hline & $\mathrm{C} 116 \mathrm{~T}$ & $21 \pm 4.1 *$ & $2.5 \pm 0.4^{*}$ & $1.3 \pm 0.1$ & $2.3 \pm 0.4$ & $4.9 \pm 0.5^{*}$ \\
\hline & C116A & $27 \pm 6.5^{*}$ & $5.9 \pm 1.6^{*}$ & $1.0 \pm 0.1$ & $2.2 \pm 0.3$ & $4.7 \pm 0.7 *$ \\
\hline & $\mathrm{C} 165 \mathrm{~T}$ & $45 \pm 3.8 *$ & $9.2 \pm 2.7^{*}$ & $1.6 \pm 0.2$ & $2.6 \pm 0.4$ & $3.4 \pm 0.3^{*}$ \\
\hline & $\mathrm{C} 165 \mathrm{~A}$ & $42 \pm 8.9^{*}$ & $5.6 \pm 1.8^{*}$ & $1.5 \pm 0.2$ & $2.3 \pm 0.6$ & $5.7 \pm 0.5^{*}$ \\
\hline & $\mathrm{C} 116 \mathrm{~T}+\mathrm{C} 165 \mathrm{~T}$ & $57 \pm 19 *$ & $13 \pm 4.4^{*}$ & $1.8 \pm 0.4$ & $3.0 \pm 0.5$ & $4.6 \pm 0.5^{*}$ \\
\hline \multirow[t]{5}{*}{$S S 2$} & $\mathrm{C} 126 \mathrm{~T}$ & n. d. & $5.3 \pm 1.7^{*}$ & $0.2 \pm 0.02 *$ & $0.6 \pm 0.1 *$ & $4.5 \pm 0.3^{*}$ \\
\hline & C126A & $24 \pm 5.6^{*}$ & $3.7 \pm 1.0 *$ & $0.6 \pm 0.1^{*}$ & $2.0 \pm 0.5$ & $6.4 \pm 0.6^{*}$ \\
\hline & $\mathrm{C} 149 \mathrm{~T}$ & n. d. & $5.6 \pm 2.6^{*}$ & $0.2 \pm 0.07 *$ & $0.9 \pm 0.1 *$ & $8.0 \pm 0.8 *$ \\
\hline & C149A & n. d. & $3.1 \pm 0.9^{*}$ & $0.1 \pm 0.02 *$ & $0.7 \pm 0.1^{*}$ & $9.3 \pm 1.3^{*}$ \\
\hline & $\mathrm{C} 126 \mathrm{~T}+\mathrm{C} 149 \mathrm{~T}$ & $65 \pm 24 *$ & $10+2.4 *$ & $0.6 \pm 0.3^{*}$ & $1.7 \pm 0.4^{*}$ & $7.4 \pm 0.6^{*}$ \\
\hline \multirow[t]{5}{*}{$S S 3$} & $\mathrm{C} 132 \mathrm{~T}$ & $8.2 \pm 2.1$ & $0.9 \pm 0.1$ & $1.3 \pm 0.2$ & $2.4 \pm 0.4$ & $22 \pm 1.0$ \\
\hline & C132A & $14 \pm 4.1$ & $1.8 \pm 0.5^{*}$ & $0.3 \pm 0.1 *$ & $0.8 \pm 0.2 *$ & $10 \pm 0.6^{*}$ \\
\hline & $\mathrm{C} 159 \mathrm{~T}$ & $12 \pm 4.1$ & $1.2 \pm 0.5$ & $0.8 \pm 0.1^{*}$ & $2.3 \pm 0.3$ & $20 \pm 1.5$ \\
\hline & C159A & $5.1 \pm 1.4$ & $0.9 \pm 0.4$ & $0.9 \pm 0.2$ & $1.7 \pm 0.4$ & $20 \pm 1.7$ \\
\hline & $\mathrm{C} 132 \mathrm{~T}+\mathrm{C} 159 \mathrm{~T}$ & $5.3 \pm 0.8$ & $0.8 \pm 0.3$ & $1.3 \pm 0.1$ & $2.2 \pm 0.4$ & $22.4 \pm 1.3$ \\
\hline \multirow[t]{6}{*}{ SS4 } & $\mathrm{C} 217 \mathrm{~T}$ & $7.2 \pm 2.9$ & $0.9 \pm 0.1$ & $0.9 \pm 0.2$ & $1.5 \pm 0.2 *$ & $18 \pm 1.5$ \\
\hline & C217A & n. d. & n. d. & $0.1 \pm 0.05 *$ & $0.3 \pm 0.1 *$ & $6.7 \pm 0.5^{*}$ \\
\hline & $\mathrm{C} 217 \mathrm{R}$ & $9.2 \pm 2.3$ & $1.9 \pm 0.8$ & $1.2 \pm 0.3$ & $2.7 \pm 0.4$ & $15 \pm 6.3$ \\
\hline & $\mathrm{C} 227 \mathrm{~T}$ & n. d. & n. d. & $0.1 \pm 0.05 *$ & $0.2 \pm 0.05^{*}$ & $4.9 \pm 0.9 *$ \\
\hline & $\mathrm{C} 227 \mathrm{~A}$ & n. d. & n. d. & $0.1 \pm 0.04 *$ & $0.3 \pm .0 .1 *$ & $6.3 \pm 1.1 *$ \\
\hline & $\mathrm{C} 217 \mathrm{~T}+\mathrm{C} 227 \mathrm{~T}$ & n. d. & n. d. & $0.2 \pm 0.03 *$ & $0.3 \pm 0.1 *$ & $7.7 \pm 1.8 *$ \\
\hline \multirow[t]{5}{*}{ SS5 } & $\mathrm{C} 261 \mathrm{~T}$ & n. d. & $1.1 \pm 0.4$ & $0.3 \pm 0.1 *$ & $0.9 \pm 0.2 *$ & $22 \pm 1.6$ \\
\hline & C261A & $5.5 \pm 1.7$ & $0.8 \pm 0.4$ & $1.6 \pm 0.3$ & $2.7 \pm 0.7$ & $19 \pm 2.0$ \\
\hline & $\mathrm{C} 270 \mathrm{~T}$ & $3.4 \pm 1.8$ & $0.9 \pm 0.3$ & $0.5 \pm 0.2 *$ & $1.6 \pm 0.2 *$ & $31 \pm 3.2 *$ \\
\hline & $\mathrm{C} 270 \mathrm{~A}$ & n. d. & $1.0 \pm 0.2$ & $0.2 \pm 0.03^{*}$ & $1.1 \pm 0.2 *$ & $19 \pm 1.4$ \\
\hline & $\mathrm{C} 261 \mathrm{~T}+\mathrm{C} 270 \mathrm{~T}$ & $1.7 \pm 0.8$ & $0.3 \pm 0.1 *$ & $0.4 \pm 0.1^{*}$ & $1.4 \pm 0.4^{*}$ & $26 \pm 1.5$ \\
\hline
\end{tabular}

suggested previously that trafficking of P2X1R to the plasma membrane is reduced by disruption of the SS5 bond, accounting for the smaller $\mathrm{I}_{\max }$ value (Ennion and Evans 2002). However, the EGFP-tagged A-mutants of the SS5 cysteines were localized to the membrane region. Furthermore, there was a large rescue effect of IVM on ATP-induced current amplitude, demonstrating the functional presence of receptors at the plasma membrane.
In our experiments, the lower $I_{\max }$ values for four of the five SS5 mutants could also reflect their preference for the closed state of the channel.

Taken together, experiments with SS mutants suggest the receptor specificity in the requirement for the $\mathrm{SS}$ bonds for the proper receptor function. In P2X1R none of these bonds per se is essential for receptor function (Ennion and Evans 2002). For P2X2R, however, 
the SS1-4 bonds are individually needed for the proper operation of the receptor (Ennion and Evans 2002). For the P2X4R, the SS3 bond appears not to be critical as the double SS3 mutant and three of four single mutants were fully functional. The SS4 bond is absent in the simple eukaryote Ostreococcus, and SS2, SS3, SS4 and SS5 bonds are absent in Dictyostelium (Jarvis and Khakh 2009, Surprenant and North 2009). These receptors are functional, but a high concentration of agonist is required for their activation. Thus, it is reasonable to conclude that formation of the SS4 and SS5 bonds was an important step in the evolution of $\mathrm{P} 2 \mathrm{XR}$ proteins.

Our results also showed that not all single residue SS mutants behaved similarly and comparably to the double mutants. For example, the maximum current amplitude was much lower for three of four single point SS2 mutants (C132T, C159A, and C159T), compared with the SS2 double mutant, and there was a decrease in ATP sensitivity and $I_{\max }$ in cells expressing the C132A, mutant, whereas the receptor function was not affected by double SS3 mutants. Similarly, the P2X4R function was not affected when the cysteine 217 was replaced with threonine or arginine. In addition, mutant C261A showed properties comparable to the WT receptor, in contrast to double and three single SS5 mutants. These results indicate that individual cysteines forming the SS2, SS3, SS4 and SS5 bonds or their substitutes might also play a role in receptor function independently of the loss in bonding, probably by generating new interactions that might impair and/or preserve the structure of the protein necessary for agonist binding and/or channel gating.

The crystal structure of zebrafish P2X4R (Kawate et al. 2009) provides some rationale for the specific roles of the SS1-5 disulfide bonds in P2XR function (Fig. 4). It shows that a long turn of 13 amino acids starting from cysteine 165 (in green) could inhibit ATP binding by covering the ligand binding site if liberated by disruption of the SS1 bond. Disruption of the SS2 could also change the shape or size of the ATP binding pocket, as well as the head-to-body interface. Another long turn of 14 amino acids, called the dorsal fin (in green), preceding cysteine 217 could play a similar role in the opposite half of the predicted ATP binding site if liberated by disruption of the SS4 bond. Thus, the inhibitory effect of the SS4 double mutant on receptor function could be explained by changes in the leftflipper-to-dorsal-fin interface, which is one of three major subunit-subunit contacts suggested to be important for receptor function (Kawate et al. 2009). The model also

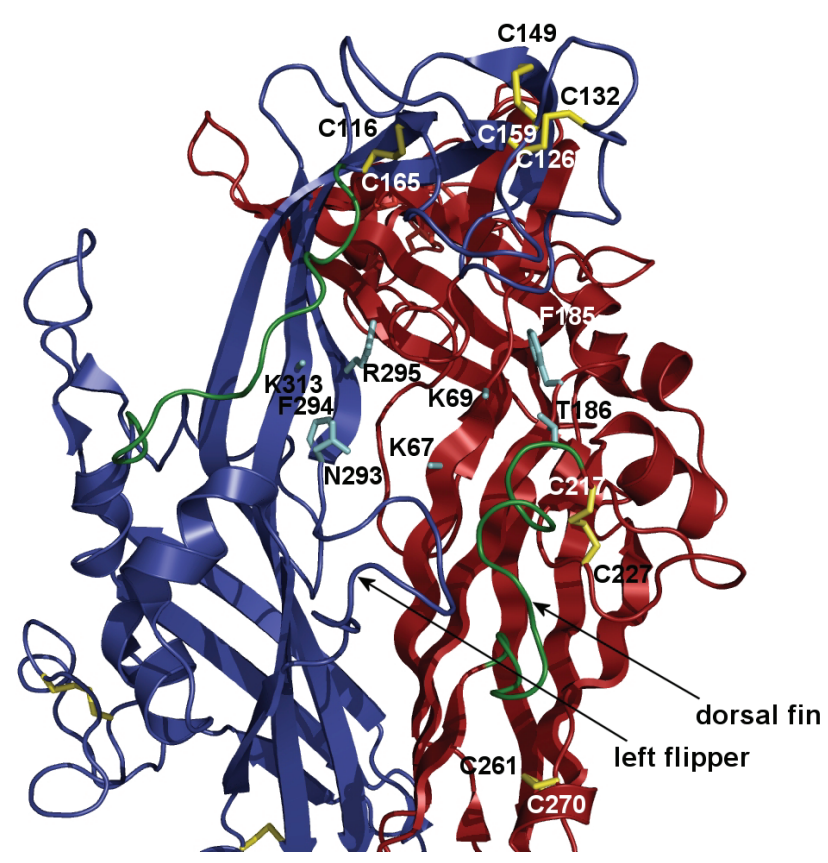

Fig. 4. Model of two $z P 2 X 4.1 R$ subunits with the putative ATP binding site (cyan) and position of conserved disulfide bonds (yellow) based on the crystal structure data (Kawate et al. 2009). The following conserved residues (rat P2X4R numbering) are predicted to form the ATP binding site at the interface between two subunits: K67, K69, F185 and T186 in the red subunit and N293, F294, R295 and K313 in the blue subunit (Ennion et al. 2000, Jiang et al. 2000, Roberts and Evans 2004, 2006, Yan et al. 2006, Zemková et al. 2007). The third subunit was removed for clarity but would be behind the two shown. Two turns (green) might be relaxed by disruption of the SS1 and SS4 bonds. The turn that precedes the $\mathrm{C} 217$ residue is termed the dorsal fin and is facing the left flipper (blue turn). This interface may involve interaction between subunits because earlier experiments showed that alanine substitution of single residue in the left flipper of the P2X3R (aspartate 280, the rat P2X4 numbering) significantly changes kinetics of receptor resensitization (Fabbretti et al. 2004).

shows that the SS5 bond is located relatively far from the putative ATP binding site but close to the extracellular vestibule above the TM domains, supporting its role in channel gating.

\section{Conflict of Interest}

There is no conflict of interest.

\section{Acknowledgements}

This study was supported by the Internal Grant Agency of Academy of Sciences (Grants No. IAA500110910), the Grant Agency of the Czech Republic (305/07/0681), the Academy of Sciences of the Czech Republic (Research Project No. AVOZ 50110509), the Centrum for Neuroscience (Research Project No. LC554) and the Intramural Research Program of the NICHD, NIH. 


\section{References}

CLYNE JD, WANG LF, HUME RI: Mutational analysis of the conserved cysteines of the rat P2X2 purinoceptor. J Neurosci 22: 3873-3880, 2002.

CODDOU C, ACUNA-CASTILLO C, BULL P, HUIDOBRO-TORO JP: Dissecting the facilitator and inhibitor allosteric metal sites of the P2X4 receptor channel: critical roles of CYS132 for zinc potentiation and ASP138 for copper inhibition. J Biol Chem 282: 36879-36886, 2007.

ENNION S, HAGAN S, EVANS RJ: The role of positively charged amino acids in ATP recognition by human P2X1 receptors. J Biol Chem 275: 29361-29367, 2000.

ENNION SJ, EVANS RJ: Conserved cysteine residues in the extracellular loop of the human P2X1 receptor form disulfide bonds and are involved in receptor trafficking to the cell surface. Mol Pharmacol 61: 303-311, 2002.

FABBRETTI E, SOKOLOVA E, MASTEN L, D'ARCO M, FABBRO A, NISTRI A, GINIATULLIN R. Identification of negative residues in the P2X3 ATP receptor ectodomain as structural determinants for desensitization and the $\mathrm{Ca}^{2+}$ sensing modulatory sites. J Biol Chem 279: 53109-53115, 2004.

FRIDAY SC, HUME RI: Contribution of extracellular negatively charged residues to ATP action and zinc modulation of rat P2X2 receptors. J Neurochem 105: 1264-1275, 2008.

JARVIS MF, KHAKH BS: ATP-gated P2X cation-channels. Neuropharmacology 56: 208-215, 2009.

JELÍNKOVÁ I, YAN Z, LIANG Z, MOONAT S, TEISINGER J, STOJILKOVIC SS, ZEMKOVÁ H: Identification of P2X4 receptor-specific residues contributing to the ivermectin effects on channel deactivation. Biochem Biophys Res Commun 349: 619-625, 2006.

JIANG LH, RASSENDREN F, SURPRENANT A, NORTH RA: Identification of amino acid residues contributing to the ATP-binding site of a purinergic P2X receptor. J Biol Chem 275: 34190-34196, 2000.

KAWATE T, MICHEL JC, BIRDSONG WT, GOUAUX E: Crystal structure of the ATP-gated P2X4 ion channel in the closed state. Nature 460: 592-598, 2009.

KHAKH BS, NORTH RA: P2X receptors as cell-surface ATP sensors in health and disease. Nature 442: 527-532, 2006.

KHAKH BS, PROCTOR WR, DUNWIDDIE TV, LABARCA C, LESTER HA: Allosteric control of gating and kinetics at P2X4 receptor channels. J Neurosci 19: 7289-7299, 1999.

NICKE A, BAUMERT HG, RETTINGER J, EICHELE A, LAMBRECHT G, MUTSCHLER E, SCHMALZING G: $\mathrm{P} 2 \mathrm{X} 1$ and $\mathrm{P} 2 \mathrm{X} 3$ receptors form stable trimers: a novel structural motif of ligand-gated ion channels. EMBO J 17: 3016-3028, 1998.

NORTH RA: Molecular physiology of P2X receptors. Physiol Rev 82: 1013-1067, 2002.

PRIEL A, SILBERBERG SD: Mechanism of ivermectin facilitation of human P2X4 receptor channels. J Gen Physiol 123: 281-293, 2004.

RASSENDREN F, BUELL G, NEWBOLT A, NORTH RA, SURPRENANT A: Identification of amino acid residues contributing to the pore of a P2X receptor. Embo J 16: 3446-3454, 1997.

ROBERTS JA, EVANS RJ: ATP binding at human P2X1 receptors. Contribution of aromatic and basic amino acids revealed using mutagenesis and partial agonists. $J$ Biol Chem 279: 9043-9055, 2004.

ROBERTS JA, EVANS RJ: Contribution of conserved polar glutamine, asparagine and threonine residues and glycosylation to agonist action at human P2X1 receptors for ATP. J Neurochem 96: 843-852, 2006.

SILBERBERG SD, LI M, SWARTZ KJ: Ivermectin interaction with transmembrane helices reveals widespread rearrangements during opening of P2X receptor channels. Neuron 54: 263-274, 2007.

SURPRENANT A, NORTH RA: Signaling at purinergic P2X receptors. Annu Rev Physiol 71: 333-359, 2009.

TOULME E, SOTO F, GARRET M, BOUE-GRABOT E: Functional properties of internalization-deficient P2X4 receptors reveal a novel mechanism of ligand-gated channel facilitation by ivermectin. Mol Pharmacol 69: 576-587, 2006.

YAN Z, LIANG Z, OBŠIL T, STOJILKOVIC SS: Participation of the Lys313-Ile333 sequence of the purinergic P2X4 receptor in agonist binding and transduction of signals to the channel gate. J Biol Chem 281: 32649-32659, 2006. 
YAN Z, LIANG Z, TOMIC M, OBŠIL T, STOJILKOVIC SS: Molecular determinants of the agonist binding domain of a P2X receptor channel. Mol Pharmacol 67: 1078-1088, 2005.

YI CL, LIU YW, XIONG KM, STEWART RR, PEOPLES RW, TIAN X, ZHOU L, AI YX, LI ZW, WANG QW, LI $\mathrm{CY}$ : Conserved extracellular cysteines differentially regulate the inhibitory effect of ethanol in rat P2X4 receptors. Biochem Biophys Res Commun 381: 102-106, 2009.

ZEMKOVÁ H, YAN Z, LIANG Z, JELÍNKOVÁ I, TOMIC M, STOJILKOVIC SS: Role of aromatic and charged ectodomain residues in the P2X4 receptor functions. J Neurochem 102: 1139-1150, 2007. 